\title{
Optics in the atmosphere: what happened in the skies of St. Petersburg on 18 June 1790
}

\author{
Robert Greenler
}

Robert G. Greenler, "Optics in the atmosphere: what happened in the skies of St. Petersburg on 18 June 1790," Proc. SPIE 1603, Education in Optics, (1 March 1992); doi: 10.1117/12.57868 


\title{
Invited Paper
}

Optics in the Atmosphere -- What Happened in the Skies of

St. Petersburg on 18 June, 1790?

Robert Greenler

Department of Physics

University of Wisconsin-Milwaukee

Milwaukee, WI 53201

\begin{abstract}
There is a published, eyewitness record of a complex display of circles, arcs, and spots of light in the sky over St. Petersburg in 1790. The display can be explained as resulting from the reflection and refraction of light by small, air-borne ice crystals.
\end{abstract}

\section{INTRODUCTION}

On 18 June, 1790 Tobias Lowitz saw an amazing collection of halos, streaks, and spots in the sky of St. Petersburg. His drawing of what he saw was published ${ }^{1}$ in 1794, along with a brief commentary on the appearance of the effects, but with no explanation of their origins. (See Fig. 1) How can we go about understanding such a complicated display? This paper briefly indicates an approach taken by the author and his associates to understand the phenomena documented by this drawing. ${ }^{2}$ A more complete description is given in the book "Rainbow, Halos, and Glories". 3 The few photos reproduced in black and white here are pale representations of the original color slides ${ }^{4}$ used as the "data" with which to compare the computer simulations.

When the job at hand is to understand a very complicated set of observations, the obvious place to start is by breaking the problem into simpler pieces. Some of the elements in Lowitz' drawing have long been recognized as effects produced by minute ice crystals falling through the air. The collection of many such crystals constitute an ice cloud or an ice fog (depending on the location of the observer). Progress on understanding the observations of Lowitz was made by understanding, one at a time, the origins of the individual components of the display. (In this presentation I may somewhat misrepresent the motivation for the work as being directed to understand Lowitz' observation. Actually, the motivation was to understand the beautiful effects seen in the sky and the understanding of the St. Petersburg puzzle was one of the side benefits that resulted.)

\section{HALOS AND SUN DOGS}

I will discuss effects that can result from the two types of ice crystals shown in Fig. 2. Actually both are right, hexagonel prisms. I will refer to the flat, hexagonal form as a plate crystal and the long, columnar form as a pencil crystal (because of its resemblance to a wooden pencil -- before sharpening). Both of these forms have been collected, photographed, and are known commonly to exist in the sky. They give rise to most of the observed ice crystal halos and arcs. 
I will start with the most common effect that results from ice crystals in the air: the halo that you can sometime see around the sun or moon. (Fig. 3) Sometimes you see only a part of the halo, and sometimes you see a very bright halo when the sky is nearly clear and you might think that there are few ice crystals present. The halo is evidence that well-formed crystals are present.

Fig. 4 shows a light ray passing through a hexagonal crystal. The dotted lines extending the sides of the hexagon show that the ray is deviated exactly as if it were passing through a $60^{\circ}$ prism. The deviation angle of $22^{\circ}$, shown in the figure, results from the $60^{\circ}$-prism angle and the index of refraction of ice. The deviation, however, depends on the orientations of the prism. If the prism is rotated either clockwise or counterclockwise from the position shown, the deviation increases. The figures illustrates the minimum-deviation angle, which occurs when the light ray makes the same angle with both faces of the prism. Any other orientation of the prism will result in a greater deviation angle. One consequence of this minimum-deviation angle is that if we have a large number of crystals, with different orientations, we would see that there are more rays deviated by approximately $22^{\circ}$ than by any other amount. Small changes in the orientation of the prism, near this minimum-deviation orientation, don't change the deviation angle of the light by very much.

Think of where you would look in the sky (filled with randomly oriented ice crystals that are illuminated by the parallel rays of light from the distant sun) to see an ice crystal that is deviating a light ray by an angle of $22^{\circ}$ and sending it to your eye. Fig. 5 shows the answer. You would look in a direction that is $22^{\circ}$ away from the sun. That describes not one direction in the sky, but a circle around the sun, a circle with an angular radius of $22^{\circ}$. So, it becomes obvious why the halo is called "the 22-degree halo". As you know, different colors of light are deviated by different amounts as they go through a prism. Red light is deviated by the smallest angle and, hence, the red circle is slightly smaller than the other colors and we usually see the halo with a red inner edge.

So, we understand that the 22-degree halo results from light rays passing through a 60-degree prism of ice. In this hexagonal prism (Fig. 2) there are also some $90^{\circ}$ angles. Light can pass through a $90^{\circ}$ prism, which has a minimumdeviation angle of $46^{\circ}$. The same kind of argument that explains the source of the 22-degree halo would predict that there should also be a 46-degree halo. The 22degree halo is a BIG halo, but the 46-degree halo is a REALLY BIG halo! It is not seen as often as the 22-degree halo but even when it is present, I think that it often is not observed, just because it is hard to identify something that is so big. Fig. 6 show a fisheye (wide angle) photo of the two halos around the sun. In Fig. 3 you can see the (nearly) complete 22-degree halo. The light from the sides of the halo would be deviated to your eyes by plate crystals, for example, with their axes nearly, vertical, whereas light from the top or bottom of the halo would be sent to your eyes by crystals with their axes roughly horizontal. In order to get the complete halo, crystals with all orientations must be present in the air. Sometimes, however, the ice crystals do not tumble randomly as they fall. A plate crystal, shaped like this model, whose diameter is a fraction of a millimeter, will tend to become orientated as it falls in still air. The orientation is (perhaps surprisingly) such as to maximize the air resistance as it falls. A plate crystal of the appropriate dimensions falls flat, like a spreadeagle sky diver, with its axis nearly vertical and its large faces nearly horizontal. 
How is the appearance of the halo affected by having a skyful of crystals with this orientation? You would see the sides of the halo but not the top or the bottom. And, in fact, you can rather commonly see this effect! Fig. 7 shows a faint 22-degree halo from a layer of randomly oriented crystals and bright spots on the halo on either side of the sun, resulting from a population of well oriented plate crystals. In English mythology they are called "false suns" or "mock suns" or "sun dogs".

Although the photo of Fig. 7 was taken in a somewhat exotic place, 22-degree halos and sun dogs are seen rather commonly (by that small fraction of people who look for them) over a large portion of the earth. I recently spend a year in Malaysia, a few degrees north of the equator. I don't recall ever seeing a report of ice-crystal halos from Malaysia but I saw halos or arcs on nearly 30 days during the year. People, who think that these effects are rare, probably don't look for them.

\section{TANGENT ARCS TO THE $22^{\circ}$ HALO}

Frequently there is an intensification of the light pattern at the top of the halo. It usually takes the form of an arc that is tangent to (touching) the halo, although it sometimes curves away from the halo and sometimes toward it. See the photos in Fig. 8.) What can be the cause of this arc? To get light from the top of the halo refracted down to your eyes, crystals would have to be oriented with their axes horizontal. Plate crystals do not orient that way but the pencil crystals do. But how can we know what patterns of light would be caused by a large group of oriented pencil crystals? We answered that question using a computer.

In principle it is not difficult to calculate how light changes its direction in going through an ice prism. We have only to apply the law of refraction (Snel1's Law) when the ray enters the prism and when it leaves. We use three angles to specify the orientation of the ice prism; then calculate the direction of a light ray from the sun after it passes through the prism; and finally calculate where in space a crystal with that orientation would send light to your eye. On a piece of paper we have the computer plot a point, representing the location of an ice crystal that would "light up". If we repeat this calculation a hundred thousand times for a hundred thousand different ice-crystal orientations, the resulting dot diagram shows us the light pattern in the sky that would be produced by such a collection of ice crystals. We started off with the simplest distribution. We considered the crystal axis to be exactly horizontal, with a random rotation about that axis and a random direction of that axis in the horizontal plane. We considered only rays that passed through alternate side faces as shown in Fig. 4.

Typical results are shown on the left side of Fig. 8, for four different elevations of the sun. The horizon is represented by the heavy horizontal line. The inner edge of the 22-degree halo is represented by the circle. The dots show the light pattern that we predict for this collection of ice crystals. For the sun on the horizon (elevation of $0^{\circ}$ ) the predicted vee-shaped arc matches quite well the arc on the adjacent photo with the sun on the horizon. As the sun elevation increases, the upper tangent arc flattens. At $20^{\circ}$ the prediction matches well the photo with the sun at the same altitude. At $30^{\circ}$, the arc is predicted to be a nearly horizontal band (where it has great enough intensity to be seen). The adjacent photo shows only one arc -- the 22-degree halo is not visible. Apparently all the ice crystals were oriented pencils, giving no trace 
of the circular halo but only the horizontal band, in fair agreement with the computer simulation. Notice that the simulations predict a lower tangent arc at the bottom of the halo. In the first two examples, this arc would be below the horizon, but could be seen from an airplane flying over an ice cloud.

When the sun is at an elevation greater than about $40^{\circ}$, an interesting, change takes place. The upper and lower arcs join at the sides to form a complete halo, encircling the 22-degree halo. It is called the circumscribed halo and is shown in the simulation for $50^{\circ}$ in Fig. 7. Fraser's adjacent photo shows the corresponding reality, produced by the laws of Nature.

We have many more photographs of the upper and lower tangent arcs and the circumscribed halo for a wide variety of sun elevations. And we have computer simulations that match them quite well.

What do we conclude from all of this? The exercise is really a rather nice illustration of how the scientific method (sometimes) works. We saw these arcs in the sky and wondered what caused them. We made the guess (hypothesis) that they might result from light passing through oriented pencil crystals. Based on that idea we used our hypothesis and the computer simulation to predict how the arcs would vary with sun elevation and how they turn into the circumscribed halo at high elevations. The beautiful agreement of these predictions with the data (photographs) convince us that we have indeed found a good explanation.

\section{SOME ARCS NEAR THE 46-DEGREE HALO}

By looking again at the drawing of Fig. 1 you might see that we are making some progress on the St. Petersburg puzzle. The computer-simulation technique turned out to be a powerful tool to investigate our hunches. We could answer the question, for example, "What effect should be produced by a ray through a plate crystal following the path shown in Fig. 9, for a collection of crystals oriented so as to produce sun dogs?" The answer is shown in the right side of the figure, where the solid line represents the inner edge of the 46-degree halo. The arc, which can occur for sun elevations up to $32^{\circ}$, lies on a circle centered on the point directly overhead (the zenith). Its form -- an arc running around the zenith -- gives it the name: the circumzenithal arc. A number of phototgraphs attest to its reality.

From our success in simulating the shapes of the upper and lower tangent arcs to the 22-degree halo, and their evolution into the circumscribed halo, we have confidence that the oriented pencil crystals exist in the atmosphere. Suppose we trace rays taking paths labelled A and B (Fig. 10) through a collection of pencil crystal with horizontal axes but all other possible orientations. On the right side of Fig. 10 you see the resulting prediction for a sun elevation of $30^{\circ}$. The inner edge of 22 and 46-degree halos are shown as circles. Rays taking the "B" path produce the upper arc (supralateral arc) and the "A" rays the lower arcs on either side of the 46-degree halo (infralateral arcs). For sun elevations above $32^{\circ}$, the upper arc disappears, so for an elevation of $50^{\circ}$, the supralateral arc is missing but the infralateral arcs look quite similar to their appearance at $30^{\circ}$.

\section{THE BIG PICTURE}

These examples show how we treated one effect at a time, finding which rays, passing through what physically reasonable distribution of ice crystal orientations, produced a pattern that matchs our photographs. After we had done 
our best to match all the photographs we could find, we decided to have a try at the famous St. Petersburg display.

In that drawing, (Fig. 1), it appears that the sun is about $50^{\circ}$ above the horizon, so we did the simulation for that sun elevation.

How should we plot the results? Lowitz' drawing shows the zenith in the center of the figure so it is clearly a drawing that covers the entire sky. We plotted the results with the same kind of mapping that would be produced by my fisheye lens. It was an arbitrary choice because we did not know how Lowitz fitted his observations over the hemisphere of the sky onto a flat piece of paper. (It is the old problem that map makers have always struggled with.) Our simulation of all the relevant effects is shown in Fig. 11. The fisheye mapping covers the entire sky, with the zenith at the center, and the entire horizon is represented by the large circle enclosing the simulations. The sun elevation is $50^{\circ}$. We have included all the effects I have discussed and others. The 22 and 46-degree halos are obvious. At a sun elevation of $50^{\circ}$ we see the circumscribed halo around the 22-degree halo. For high sun elevations the sun dogs move outward, away from the 22-degree halo, as shown on both the simulation and Lowitz' drawing. A circle of light, passing through the sun and parallel to the horizon (parhelic circle) results from reflections from vertically oriented ice-crystal faces. Intensified spots on that circle, at $120^{\circ}$ in azimuth away from the sun, (the 120-degree parhelia) result from rays that are reflected twice inside the crystal before they escape the crystal.

I won't discuss all the effects that we have simulated here. Those that appear on the side of the sky sway from the sun result from rays that are reflected from some crystal face (or faces) either externally or internally. The first look shows that we have identified many of the things that Lowitz recorded. But a closer look reveals some interesting problems.

We found that if, instead of using a fisheye mapping of the simulations, we plotted the arcs seen near the sun to match the perspective of a regular photograph (or an 18th-century drawing) of that part of the sky, the results matched Lowitz' drawing better. Compare the shape of the infralateral arcs, for example, in such a plot with a part of Lowitz' drawing in Fig. 12. The match is significantly better, suggesting that Lowitz first drew the effects in the vicinity of the sun, with the natural perspective one would use to represent a small area of the sky and then added all the other things to somehow get them all shown on the flat piece of paper.

Look at Fig. 12 to see how well or poorly we have done in reproducing Lowitz' drawing. The circular arc at the top of the 46-degree halo is most certainly the circumzenithal arc. Why don't we show it on the simulation? Because that arc does not occur when the sun is higher than $32^{\circ}$ above the horizon! What about the arc that turns downward from the bottom of the 22-degree halo? We tried everything we could think of to get such an arc for a sun elevation of $50^{\circ}--$ and failed. We get a lower tangent arc (or a near-relative of that arc, called a Parry arc) that would give such an effect for a sun elevation of $30^{\circ}$, but not for the $50^{\circ}$ elevation of this picture. Then we noted one more clue to the puzzle. There are 90 degrees between horizon and zenith. The 46-degree halo has an (inner) diameter of $92^{\circ}$ and yet Lowitz shows it fitting between horizon and zenith with space to spare, both above and below it! Now we begin to understand. Lowitz says that he first saw the display at 7:30 in the morning (when the sun would have been low on the horizon). He describes the display as having attained its most 
beautiful perfection at ten o'clock in the morning (when the circumscribed halo would exist as shown in his drawing). But, at other times he saw other arcs and it finally became clear that, although he doesn't explicitly say so, he added these things -- from different sun elevations -- to this master drawing. He shows the circumzenithal arc as it would have appeared when the sun was about $20^{\circ}$ above the horizon and the lower arc touching the 22-degree halo, when the sun was, perhaps, $30^{\circ}$ above the horizon. Once we understand this, his notes, in fact, confirm it. At 7:30 in the morning he describes the arcs (of the circumscribed halo) as not yet in their perfection but slowly developing from the brilliant light at the top of the halo until at $9 \mathrm{o}^{\prime} \mathrm{clock}$ the entire circumscribed halo is formed. He describes the evolution of other arcs, which our simulation reproduces, as the sun rises.

So, two centuries after Lowitz recorded this amazing display, we can understand what he saw, but did not understand. And in the process we get some insight into the activities of the man who looked at the skies of St. Petersburg on the 18th day of June and marvelled at the beauty displayed there. Isn't that interesting?

\section{REFERENCES}

1. Tobias Lowitz, "Déscription d'un météore remarquable, observé à St. Pétersbourg le 18 Juin 1790" Nova Acta Academiae Scientiarum Imperialis Petropolitanae 8, 384 (1794).

2. The contributors to this work are listed as co-authors in the published papers cited in Ref. 3. Major contributions to the computer-simulation work described here were made by James Mallmann and James Mueller. Of course many other people have helped develop the understanding of these effects and some of these contributions are also documented in Ref. 3 .

3. R. Greenler, Rainbows, Halos, and Glories, Cambridge University Press, New York, 1980 (paperback ed. 1989).

4. Sets of slides of atmospheric optical effects (from reference 3 ) can be purchased for educational use from Mallmann Scientific Co., 20250 Jeffers Drive, New Berlin, WI 53146. 


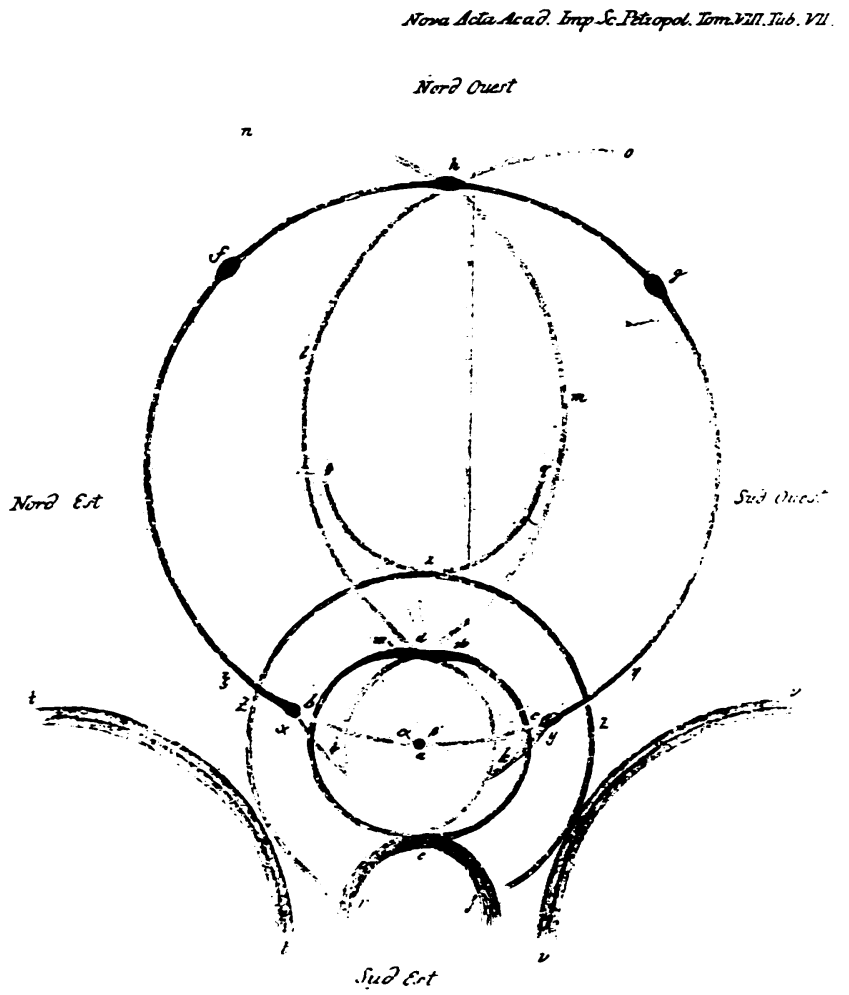

Fig. 1 Drawing by Lowitz from Ref. 1.

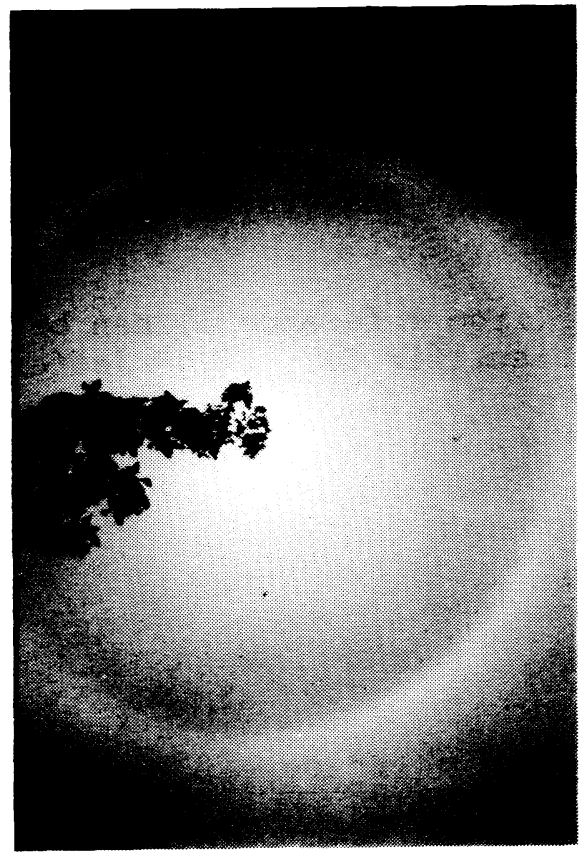

Fig. 3 22-degree, ice-crystal halo, photographed by Robert Greenler in Wisconsin.
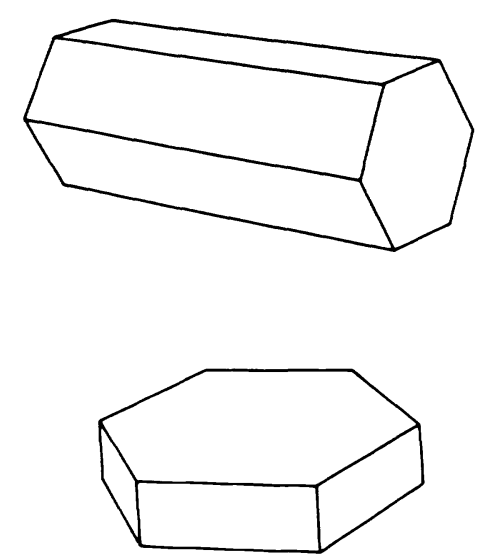

Fig. 2 The two forms of ice crystals. that produce most of the observed ice crystal halos and arcs.

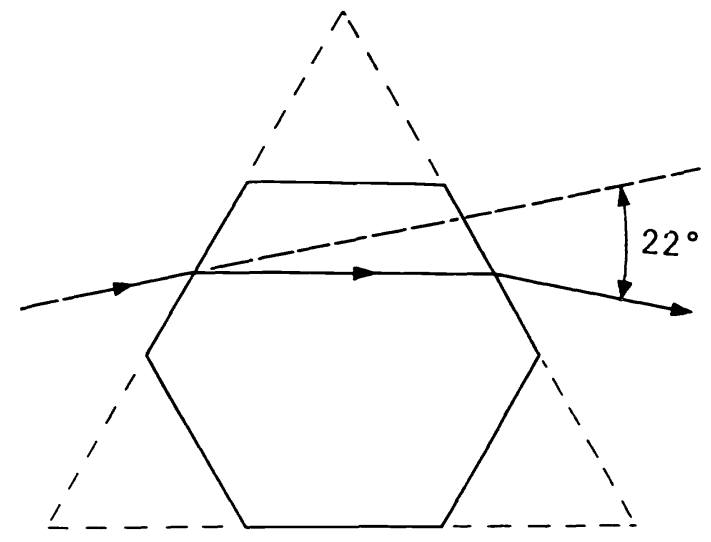

Fig 4 Path of light ray passing through alternate side faces of a hexagonal ice crystal. 


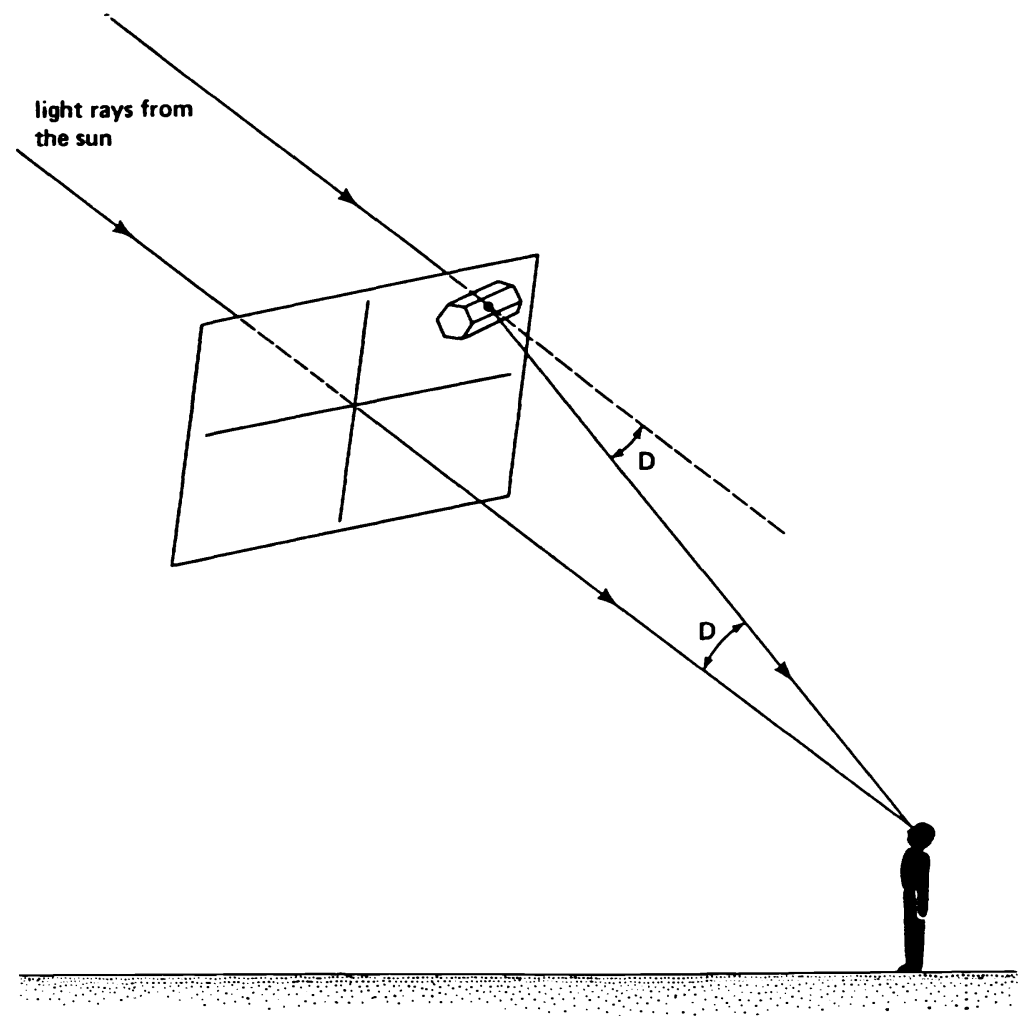

Fig. 5 To see a ray from the sun that is deviated by $22^{\circ}$, you look in a direction $22^{\circ}$ away from the sun.
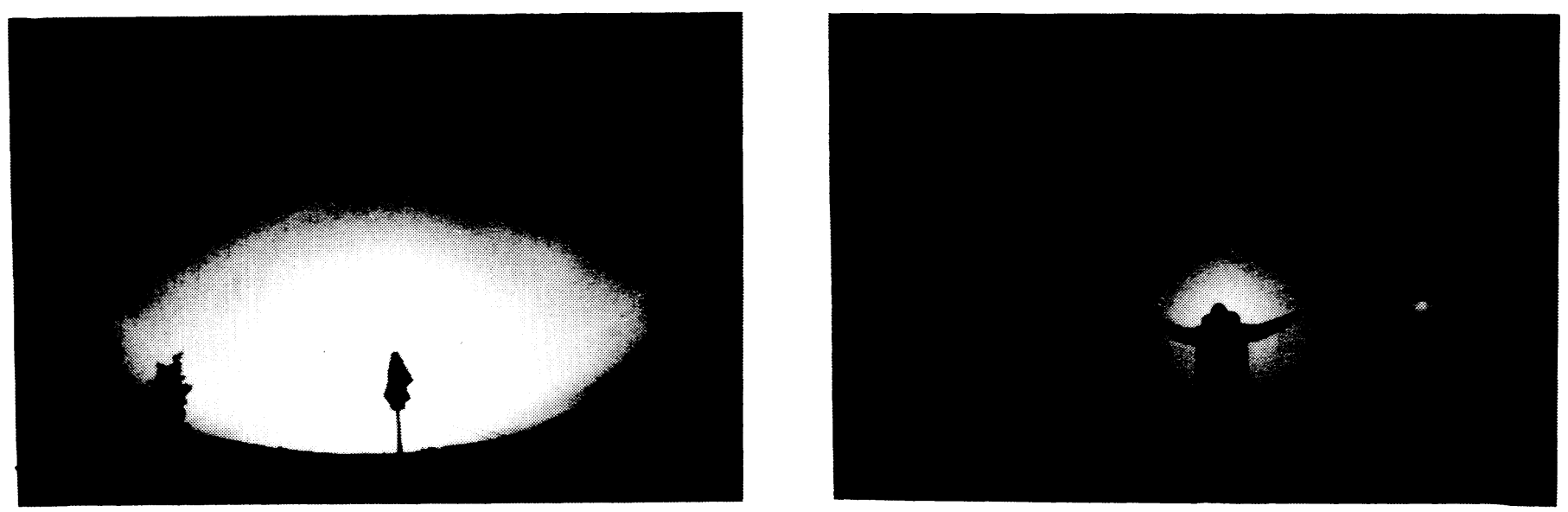

Fig. 6 The 22 and 46-degree halos, Fig. 7 Self portrait of the author, photographed by Alistair Fraser. pointing toward the sun dogs, near Point Barrow, Alaska. 

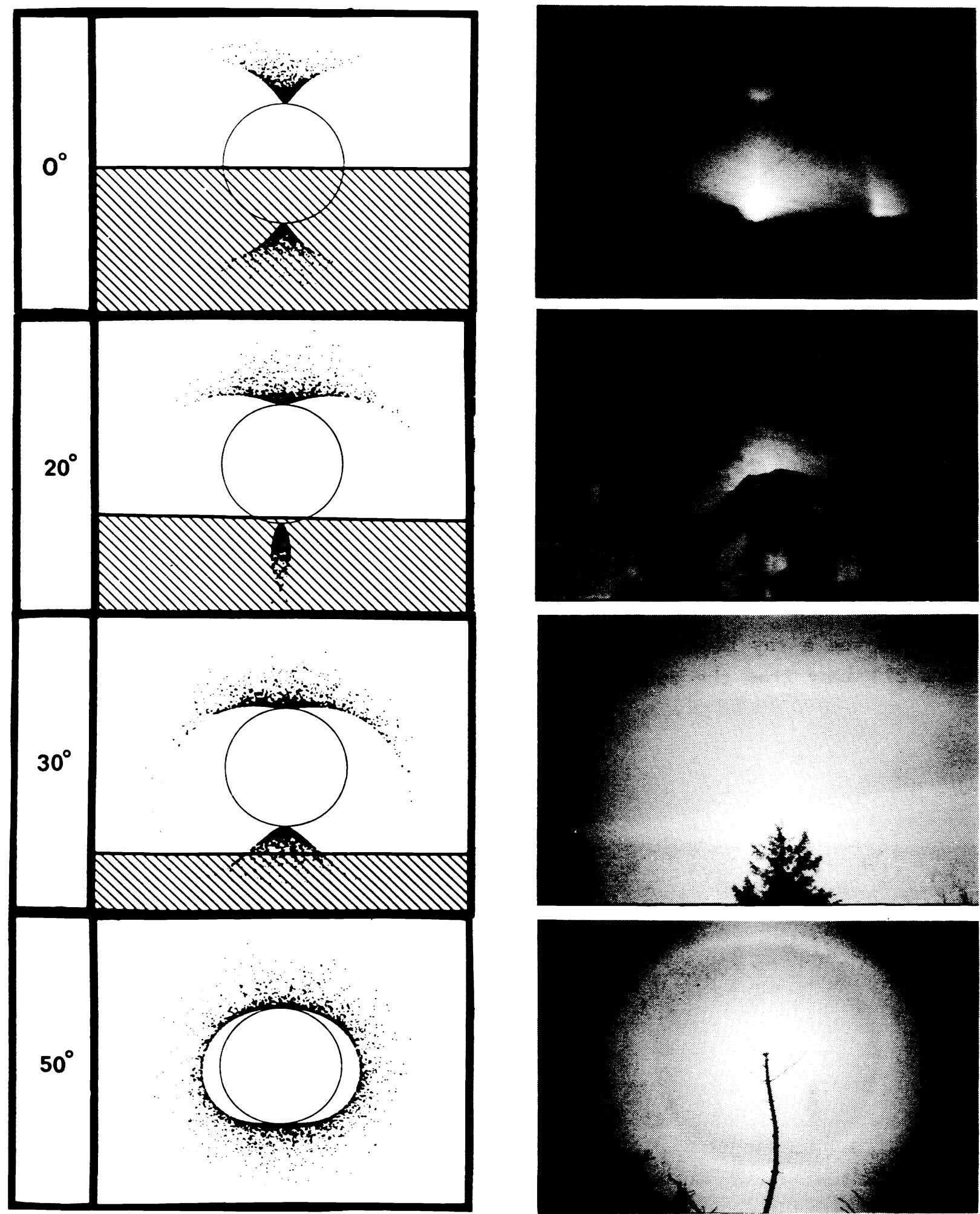

Fig. 8 On the left are computer simulations for the specified elevation of the sun above the horizon. The photos on the right were taken by (in order from the top) James Mallmann in Wisconsin, Evan Noveroski in Antarctica, Robert Greenler in Wisconsin, and Alistair Fraser in Washington. 


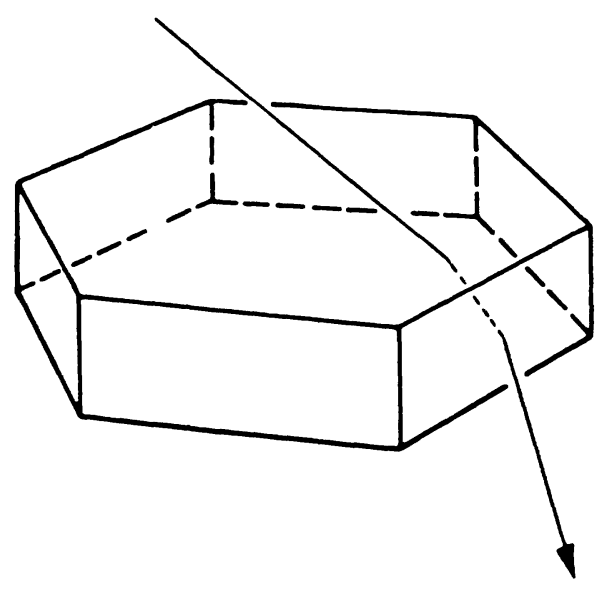

Fig. 9 At the top is shown a ray path through the crystal. To the right are the predicted (circumzenithal arc) effects resulting from such rays through a collection of plate crystals with nearly horizontal large faces but random rotational orientation about a vertical axis.

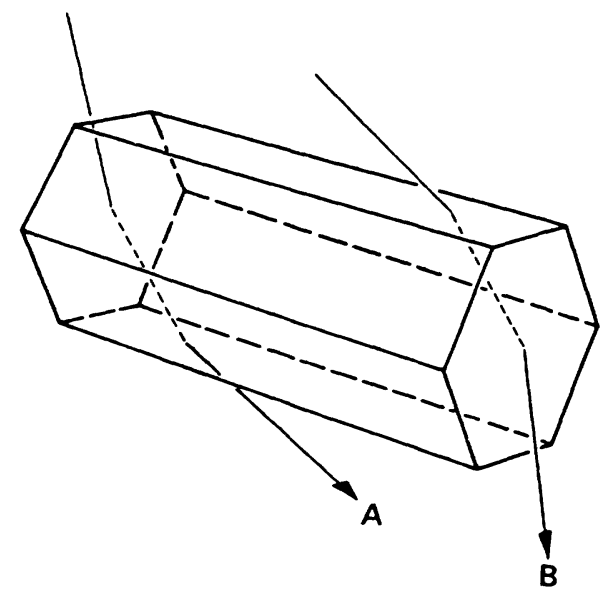

Fig. 10 Rays taking paths A and B through pencil crystals, oriented with horizontal axes, produce the supralateral arc near the top of the 46-degree halo (ray $B)$ and the infralateral arcs near the sides of the halo (ray A) for a sun elevation of $30^{\circ}$.
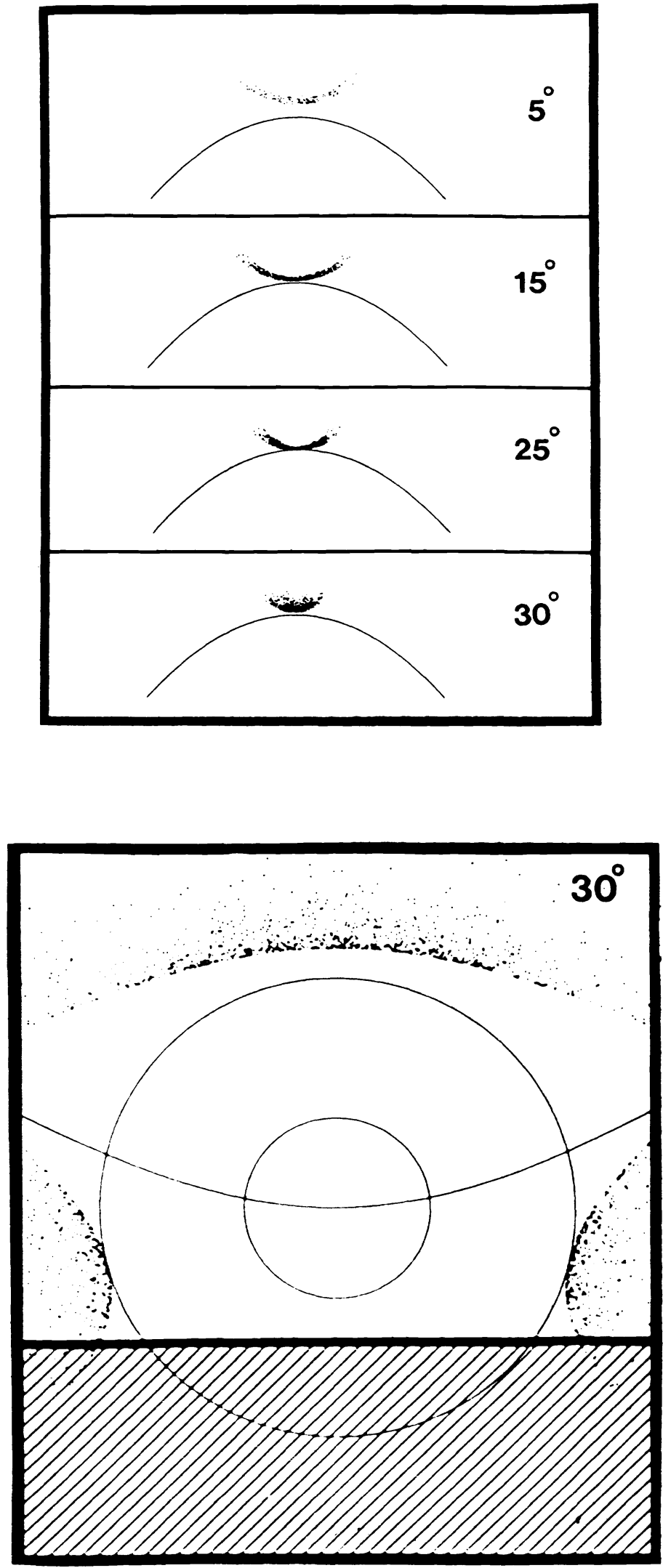


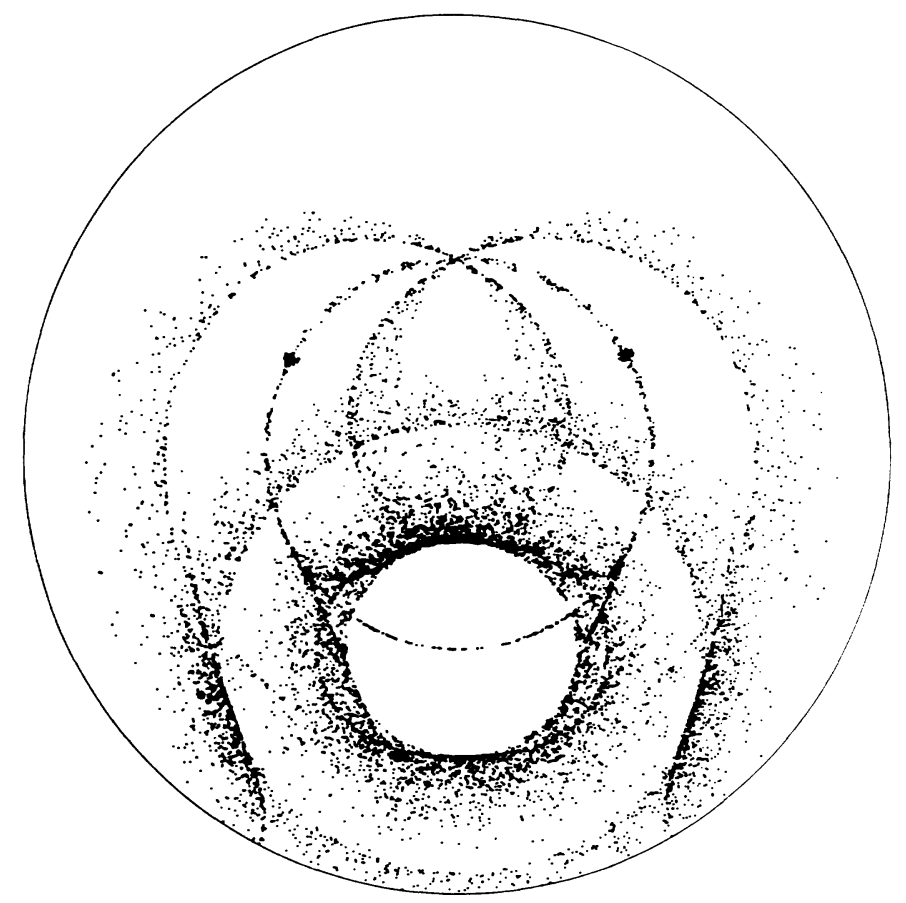

Fig. 11 Fisheye projection of the simulation of the St. Petersburg display for a sun elevation of $50^{\circ}$.

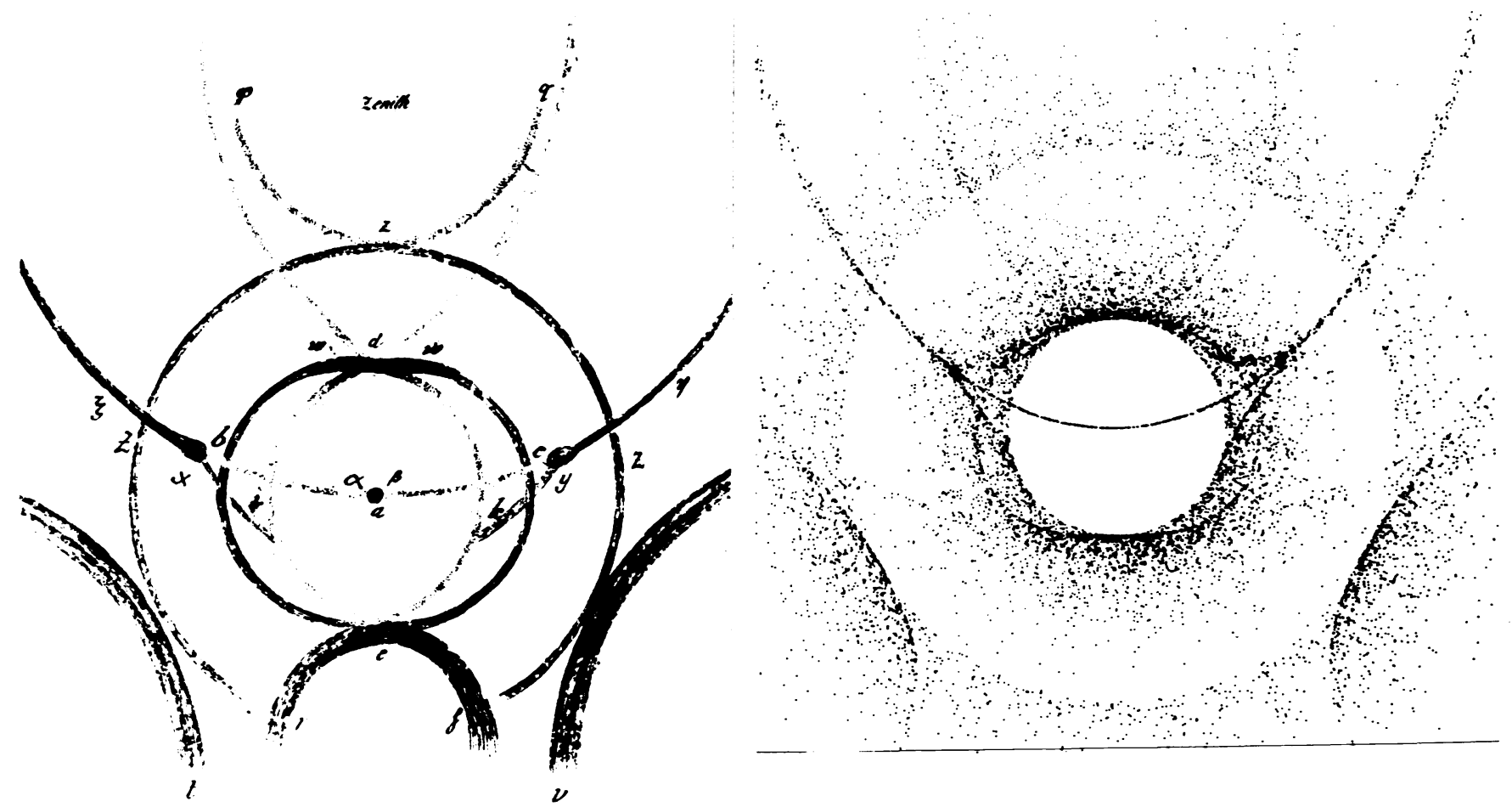

Fig. 12. A portion of Lowitz' drawing, matched by the simulation plotted with the perspective of an ordinary photograph (or 18th-century painting). 